\title{
Re-evaluating the form and communication of social robots
}

\section{The benefits of collaborating with machinelike robots}

\author{
Eleanor Sandry
}

Received: date / Accepted: date

\begin{abstract}
This paper re-evaluates what constitutes a social robot by analysing how a range of different forms of robot are interpreted as socially aware and communicative. Its argument juxtaposes a critical assessment of the development of humanlike and animal-like robotic companions with a consideration of human relations with machinelike robots in working teams. The paper employs a range of communication theories alongside ideas relating to anthropomorphism and zoomorphism in discussing human-robot interactions. Some traditions of communication theory offer perspectives that support the development of humanlike and animal-like social robots. However, these perspectives have been critiqued within communications scholarship as unethically closed to the possibilities of otherness and difference. This paper therefore reconfigures and extends the use of communication theory to explore how machinelike robots are interpreted by humans as social and communicative others. This involves an analysis of human relations with Explosive Ordnance Disposal (EOD) robots and with the robotic desk lamp, AUR. The paper positions social robotics research as important in understanding working teams containing humans and robots. In particular, this paper introduces the value of tempered anthropomorphism and zoomorphism as processes that support communication between humans and machinelike robots, while also ensuring that a sense of the otherness of the machine and respect for its non-human abilities is retained.
\end{abstract}

E. Sandry

Curtin University

Tel.: +61-8-92667436

Fax: +61-8-92663152

E-mail: e.sandry@curtin.edu.au
Keywords Communication theory - Machinelike robots $\cdot$ Anthropomorphism $\cdot$ Zoomorphism

\section{Introduction}

Although the field of social robotics is strongly linked with the placement of robots in human social spaces, social robots are sometimes thought of more generally as machines that are able to interact with one another and/or with humans in social ways [22]. When interacting with others of their own kind they may form swarm-like systems, reminiscent of organised colonies of insects such as ants or bees. Alternatively, they may interact in groups whose structure is based on an awareness of each group member as an individual, alongside a respect for particular rules and behaviours that support the success of the group as a whole. These types of group operate more like mammalian social groups than insect colonies [22][15]. Social robots that are designed to interact with one another in either of these ways take a number of forms, including machinelike (a term used in this paper to denote robots that are neither humanlike nor animal-like), animal-like and humanlike, as illustrated by the types of robots that have competed, and are currently competing, in the various categories of Robcup Soccer competition.

However, although "the media equation" theory of Byron Reeves and Clifford Nass supports the idea that humans will automatically relate to any form of robot in "fundamentally social and natural" ways, social robots, designed specifically to interact with people in human social spaces, as detailed below, are most often created in humanlike or animal-like form [44]. In addition, the research of Bartneck et al, which draws on Stanley Milgram's famous experiment in which human subjects 
participated in a process apparently involving the need to administer electric shocks to other humans, demonstrates that people's treatment of robots in such a situation does differ from their treatment of humans [4]. In particular, all people taking part were convinced to administer the highest electric shock on the scale to the robot, whereas in the original experiment some people did refuse to continue to this point [4]. The critical analysis of human-robot interactions presented in this paper therefore sets aside media equation theory to consider human communication theories, and ideas of anthropomorphism and zoomorphism, in assessing the reasons behind the focus on creating humanlike and animal-like robots in support of relations with humans. The paper then moves on to support the benefits of considering machinelike robots as social actors, by re-configuring and extending communication theory and introducing the importance of employing anthropomorphism and zoomorphism in a 'tempered' form. This type of tempered response supports people's interactions with machinelike robots, while encouraging them to retain a clear sense of the non-human abilities that such a robot can bring to a human-robot team.

The paper briefly discusses the fictional origins of robots, since these narratives identify some initial reasons for pursuing humanlike robot design, before moving on to consider real-life robots in more depth. The decision to create humanlike or animal-like robots to interact socially with humans is driven in part by the idea that something resembling a human or animal will be easy to accept quickly as a potential companion. In addition, as far as is possible, such robots are given communicative abilities that are familiar, easy for people to comprehend and to which they can respond without difficulty. This paper therefore argues that, in general, humanlike and animal-like social robot designs are based on the assumption that familiar appearance and modes of communication-whether using speech, humanlike facial expressions or the production of easily recognised and accepted animal-like reactions to human actions - are the best way to support easy and effective human-robot interactions. In particular, humanlike and animal-like social robots illustrate the idea that successful communication is not only based on what communicators have in common, but also seeks to increase the sense of commonality between them as interactions progress. From this perspective, social robots need to be able to communicate information as precisely as possible whether verbally or nonverbally, should be persuasive communicators and should be positioned within a familiar social and cultural context that is appropriate to the humans they meet. This paper therefore identifies how the assumptions of roboticists creating social robots are shaped by concerns relating to form and content: the shape of the robot and its ability to communicate information and express itself clearly are both regarded as important.

However, a critique of these assumptions about form and behaviour, and the understandings of communication upon which they depend, supports the potential of human interactions with machinelike robots. Therefore, moving beyond the overtly social goal of creating companions for people, this paper appraises the importance of personality and social positioning in situations where humans and machinelike robots work together in teams. An analysis of human responses to PackBots and Talons, robots deployed with EOD teams, illustrates how these robots are treated as social subjects, although their machinelike form and communicative abilities might be expected to introduce some difficulties. In spite of this, specific robots become highly regarded as valued team members, such that if they are damaged their repair is placed as a priority over their replacement. This occurs even though these robots are currently almost always human-controlled, as opposed to being autonomous in their operation. In the future, EOD robots are likely to support more and more autonomous capabilities. The autonomy of such robots may well increase the complexity of their interactions with humans by giving rise to situations where operators will need to trust these robots sufficiently to relinquish control to them. A further example, the robotic desk lamp, AUR, is therefore employed to consider the ramifications of this more fully. Although AUR is positioned in a laboratory, as opposed to the more dangerous environment occupied by EOD robots, a consideration of its autonomous collaboration with human participants to complete a joint task has implications for humans working with more autonomous EOD robots in the future.

The conclusion of this paper suggests that developing a social understanding of a robot with which one is required to work strengthens the human-robot team, enabling an effective use of both human and robot abilities to complete tasks in cooperative ways. This social understanding is likely to become increasingly important as the robot becomes more autonomous, since people working with such a robot must feel able to trust it to do its job, even when it is left unattended or moves out of sight or radio contact. Indeed, as robots are developed that are able to sense their surroundings and learn tasks for themselves with more accuracy, people working with them may need to allow their judgment to be re-calibrated by the robot's understanding of the environment and the task at hand, such that the team 
can operate at the peak of its potential and complete its job successfully.

\section{Social robots and familiarity in fiction and in real life}

Arguments for developing humanlike robots were first articulated in early science fiction, establishing a long tradition connecting ideas about robots with humanlike form. Karel Capek's play R. U. R. (Rossum's Universal Robots) in 1920, and Fritz Lang and Thea von Harbou's film Metropolis in 1927, both describe the creation of humanlike replacements for people at work [8][32]. Indeed, Capek's play contains the first use of the term "robot" to describe its "artificial people". These narratives link the creation of robots similar to humans in shape and size, able to communicate and be communicated with in familiar human ways, with the easy replacement of human workers in human-tailored working environments. In fiction that followed, notably that of Isaac Asimov, the creation of the perfect humanlike robot worker was also justified in more social terms through narratives such as The Bicentennial Man, which describes a robot that, amongst other changes, becomes increasingly able to express emotions [3]. In the non-fiction essay, "The Friends We Make", Asimov suggested that "if we are to take on thinking partners - or, at the least, thinking servants - in the form of machines, we will be more comfortable with them, and we will relate to them more easily, if they are shaped like humans" [3]. He extended this appraisal even further into the realm of the social when he argued that it would also "be easier to be friends with human-shaped robots than with specialized machines of unrecognizable shape" [3]. Asimov's writing therefore reinforced the idea that humanlike form and behvaiour was linked with the successful creation of a social robot, cementing this conception of the robot in the popular cultural imagination.

Similar assumptions to those considered within science fiction, regarding both form and communication in support of social human-robot interactions, are found in real-life. Although it has now been re-written, the Hanson Robotics' website expressed the desire "to model the behavior and movements of people in robots that act and react virtually indistinguishably from their human counterparts" [28]. The ultimate goal was to design robots able to evolve "into socially intelligent beings, capable of love and earning a place in the extended human family" [28]. In a more recent, and measured, statement, the RoboKind website (another company founded by David Hanson) describes the desire to "design and build a new series of social robots that allow people to interact with robots in a more natural manner and on a more personal level than ever before" [45]. Hanson, and other roboticists such as Hiroshi Ishiguro, concentrate on producing realistic humanlike robots, with the aim of making them as indistinguishable as possible from a human communicator. While Ishiguro is famous for creating his own double, Hanson has created robotic heads resembling Phillip K. Dick and Albert Einstein, as well as new individuals such as Jules. The idea that social robots should be able to communicate in a "natural manner", where this really means a natural human or humanlike manner, is also articulated by Cynthia Breazeal and Anne Foerst who argue that the best way to support human-robot interactions is through the creation of robots that can "participate in natural, human-style social exchange" [6]. Breazeal, for example, describes "a sociable robot" as "socially intelligent in a humanlike way", such that "interacting with it is like interacting with another person" [7]. In pursuing the creation of such a robot, Breazeal designed Kismet at Massachusetts Institute of Technology (MIT). As probably the first robot specifically created to be social or sociable, Kismet was designed to reproduce human facial expressions to indicate the six emotions identified by some researchers as basic to all humans: happiness, sadness, anger, fear, disgust and surprise [7][19][47]. Unlike Hanson's robots, Kismet was not meant to look exactly like a human, but rather was created as a caricature, a design decision also seen in robots developed more recently, such as Domo and Mertz also at MIT.

Although very different, the creation of animal-like social robots is also based around a sense of providing something familiar, non-threatening and easy with which to interact. Robot animals, such as PARO, have been shown to provide comfort and companionship for people in the same way that a pet animal might, and can be supported in environments or situations where live animals cannot safely be introduced [33]. People's interactions with such animal-like robots are not overly complex, and are likely to be shaped by their prior experience with owning pets or other experiences with animals [33]. Roboticists therefore overtly shape these robots' communicative abilities to mirror those of animals, using sounds and body movements to indicate expected responses to human behaviours such as stroking the robot's fur. Again, it is the familiarity of these robots' form and the content expressed through their behaviour which is understood to support their positioning as social companions to people. 


\section{Anthropomorphism, "pairing" and "harmonious behavior"}

One way to theorise human interactions with humanlike robots is to consider the ways in which their form and behaviour are designed to support a process of anthropomorphism, through which human characteristics are conferred upon the robot. Scientific discourse is generally biased against anthropomorphism, arguing that any attribution of human characteristics to nonhumans is incompatible with maintaining one's objectivity [29][21]. Indeed, Marc Bekoff has gone so far as to describe anthropomorphism as one of the "dirty words' in science" being linked with "the subjective and the personal" [5]. However, social robotics research has, for some time, been open to the idea of encouraging anthropomorphic responses in humans. In particular, Turkle et al, and Kirsten Dautenhahn's early work, argued that anthropomorphism is an important part of facilitating meaningful human-robot interactions [53][16]. For example, both the form and behaviour of the robot was understood to support people's anthropomorphic response during interactions with Kismet [53]. It seems likely that similar effects might occur when people meet the types of robots designed by Hanson and Ishiguro. Instead of creating robots that "merely take advantage of the anthropomorphizing tendency in humans", it can therefore be argued that the designers of humanlike robots overcome the potentially unsound nature of anthropomorphism by positioning it as a completely reasonable way to understand their machines [16]. A similar argument can be made for the creation of animallike robots, where these are designed to mimic their living counterparts. Here it is the idea that the robot can support a zoomorphic response that is key, with a similar justification being possible for this as being a completely reasonable way to respond to a robot such as PARO, for example.

More recently, Dautenhahn has noted that anthropomorphic responses to very humanlike robots tend to raise human expectations too high in comparison with the actual ability of the robot, suggesting that social robotics researchers may wish to focus on developing robots which use a few simplified humanlike features, as well as non-humanoid robots [14]. This reappraisal is also based on questioning whether robots can be understood to behave or communicate "naturally". Dautenhahn notes that "any behaviour of a robot will be natural or artificial, solely depending on how the humans interacting with the robot perceive it" [14]. However, as well as suggesting that "the goal of human-like robots needs to be reflected upon critically", Dautenhahn also notes the continuing prevalence of the assumption "that the the ultimate goal for designers of robots for humaninhabited environments is to develop humanoid robots, i.e. robots with a human-like shape" [14].

Analysing anthropomorphism from a phenomenological perspective offers a way to understand what drives this process, and also identifies some potential difficulties beyond that of setting aside an objective scientific stance. Phenomenology, as originally formulated by Edmund Husserl, and extended more recently by philosophers such as Emmanuel Levinas, is concerned with first-person perceptions of 'phenomena', the meanings that are developed on the basis of these experiences, and the individual explanations about the world that result. A phenomenological perspective on humanrobot interactions offers a theoretical basis from which to reconsider the value of anthropomorphic responses to robots. Husserl for example suggests that the resemblance of one body to another can be understood to support a process of what he terms "pairing" [31]. It is through pairing that a person "understands something new and unfamiliar, by analogy with something already known and familiar" [11]. In addition, the continuous demonstration of "harmonious behavior", as seen in a robot that effectively mimics human expressions, further supports a person's ability to understand that machine [11]. Clearly the linked concepts of 'pairing' and 'harmonious behavior' are useful to contextualise the way in which anthropomorphism, while it is a subjective response, is understood to work in support of "natural" and therefore meaningful human interactions with humanlike robots [6][7][45].

However, there are a number of problems with these concepts. In particular, there is the question of how close the resemblance, in form and behaviour, must be in order to support understanding between communicators. Briankle Chang asks, for example, whether it is possible for a "healthy white middle-aged bachelor" to recognise a "deformed pregnant black teenager" [11]. In terms of human-robot interactions, this raises the question of exactly what, or who, humanlike robots are designed to resemble and how this might alter communication with them. If one builds a white Caucasian male robot will this limit the range of situations within which such a robot can communicate successfully, or is any form of humanness sufficient? Husserl himself offers no means of defining the "minimal level of similarity between the two bodies being paired", and was aware of the difficulty this introduced for people wishing to theorise intersubjective relations using this concept [11].

In robotics itself, the question of how helpful it is to strive for a close resemblance between human and robot has also been raised, since encounters with very humanlike robots invoke reactions of discomfort for some 
people. This type of reaction is most often described in terms of the "uncanny valley" introduced by Masahiro Mori in 1970 [40]. With this theory Mori predicts that as the humanlike appearance of a robot increases, so does its familiarity, but there is a point at which the robot is suddenly perceived as zombielike and therefore very unattractive. Mori's terminology can be linked with Sigmund Freud's conception of the "uncanny" as "that class of the terrifying which leads back to something long known to us, once very familiar" [23]. The theory is often illustrated with a graph, showing the rise in familiarity, followed by a sudden drop, into the "uncanny valley" when the robot's humanlike nature becomes unsettling as opposed to attractive [40]. Although the graph does rise again, following its valley section, thus far it is in fictional contexts that robots have most clearly overcome people's uncanny reaction, as seen in the example of Data, the android officer from Star Trek: The Next Generation.

The focus on creating humanlike social robots demonstrates that the idea of pairing is still an attractive basis upon which to support communication. Indeed, it also goes some way to indicate that the concept does contain a considerable degree of flexibility. While robots created by Hanson and Ishiguro for example would seem to stress the importance of sharing a very similar form with humans, robots such as Kismet are clearly not designed to look as humanlike as either Ishiguro's double or Hanson's robot, Jules. The machinelike aspects of the appearance of Kismet mean that it cannot easily be categorised as white or indeed as male or female [53]. In spite of this, its cartoon-like face and ability to read people's behaviour and to behave in humanlike ways allows it to provide an easily recognisable level of humanness. In addition, although animal-like robots are designed primarily to promote a zoomorphic as opposed to an anthropomorphic response, they are still likely to be anthropomorphised by people with whom they come into contact, since such responses are also elicited in respect to animals, in particular household pets. Of course, this level of anthropomorphic response, whether to an animal or an animal-like machine, is exactly that which scientific, and scholarly discourse more generally, regards as "intellectually unsound" [29][5][21]. However, as I argue below, retaining a level of acceptance for the utility of such anthropomorphic responses may be an essential part of enabling effective human-robot interactions and teamwork.

\section{Familiarity in communication}

In broad terms, Husserl's phenomenological appraisal of the importance of commonality, both in form and behaviour, can be understood to act as "the very foundation of modern theories of communication" [11]. The assumption that humanlike robots are likely to make more effective social robots can therefore also be understood as based on particular understandings of communication that value commonality over difference. The suggestions of science fiction writers such as Asimov, as well as the design paths followed in real life - whether the realistic pathway of Hanson, for example, or the caricature illustrated by Breazeal's Kismet - are driven by the desire to make robots that are familiar because they can communicate in a "natural" manner [6][7][45]. In particular, humanlike robots are designed to communicate using humanlike channels, including voice, facial expressions and bodily gestures and movements.

On some occasions the goal for humanlike robots is to communicate accurate or helpful information directly to people. This applies, for example when ASIMO is positioned as a museum guide. Understanding the communication of a robot in these terms falls within the cybernetic tradition of theory, for which communication is linked with the clear transmission or exchange of accurate information [13]. However, a robot's communication will also often be framed in terms of a particular social situation. For example, positioning ASIMO as a museum guide reinforces the idea that the robot is an information provider, and also places further importance on the clarity and accuracy of its communication. In this case, ASIMO is required to comply with the social and cultural expectations that visitors feel are appropriate for a guide, a perspective on communication that falls within the sociocultural tradition. This tradition positions communication as a means of producing, and reproducing a shared understanding of the world [13][9].

Other robots are positioned very differently, and this has an important impact on their appearance and behaviour. For example, Kismet's cute expressive face, clear responses to praise or scolding and pre-linguistic babbling speech were chosen specifically to position this robot as an infant strongly enough to convey this understanding to people visiting the robot in its laboratory setting. People interacting with Kismet were therefore placed as caregivers, causing them to exaggerate their tones of voice when communicating with Kismet as they would do with a small child. This helped the robot to respond to people's tones of voice in the expected way, thus further supporting the child-caregiver frame for these interactions [7]. There was also a sociopsychological element to Kismet's infant-like chatter which, when taken alongside its wide-eyed face, also highlights the persuasive influence that was part of its communication style [13]. Kismet drew people in by 
craning its head and neck towards them, engaging them with its stylised expressions and baby-like babbles.

The theories of communication employed abovecybernetic, sociocultural and sociopsychological —often work in parallel to position humanlike social robots, and their human communication partners, in ways that reinforce the idea of the robot as like another human. It is clear that such perspectives on communication value the idea of emphasising commonality with humans, as opposed to highlighting the differences that a robot, as machine and not human, might possess.

\section{Critiquing ideas of commonality}

The relations between humans and humanlike and/or animal-like robots would seem to be potentially very different from human relations with technologies such as computers, described by Turkle as "evocative objects" in The Second Self [52]. Instead, Turkle argues that humanlike robots such as Kismet are better thought of as "relational artifacts", because they do not "invite projection" as is the case for evocative objects; instead, relational artifacts "demand engagement", with humanlike robots demanding that people relate to them as they would to another human [52][53]. Designing robots with humanlike and animal-like form and behaviour is therefore less about evoking a wide range of interpretations and responses, and more about facilitating one clear understanding and mode of interaction for each robot. The careful framing of robots as humalike has been discussed above, in relation to ASIMO, as well as the creations of Hanson, Ishiguro and Breazeal. The statements of Hanson and Breazeal clarify that they aim to reduce uncertainty in the interaction and the misunderstanding it might cause by designing robots with familiar forms and behaviours [7][28][45]. In particular, the intention is to make interactions with the robot easier and more meaningful for more people. This might well make sense for robots designed as social companions over the short term; however, leaving a robot's form and behaviour open to interpretation may offer increased opportunities for long-term relations to develop, simply because communication with such a machine is likely to be varied and might even be more intriguing over a longer period of time.

Another problem with adopting humanlike form and behaviour to shape human-robot interactions is the limitations that this introduces into the robot's overall design. It is difficult to create an anthropomorphous robot that is also able to use novel ways with which to sense its surroundings and to communicate; instead, such a robot is in effect limited to being like a human. From an anthropocentric perspective that views humans as the pinnacle of the evolutionary process this might be acceptable, since from this point of view being humanlike could also also be the pinnacle for robots. Indeed, the development of humanlike robots is regarded by some researchers as "the Grail" of robotics [39]. However, adopting this viewpoint overlooks both the difficulties of creating robots in humanlike form, and also the possibilities of creating robots that have very different ways from humans to view, and communicate about, the world.

From a practical point of view, regarding commonality as of prime importance means that one is less likely to meet and have the opportunity to respond openly to an other's opinions, ideas, perceptions and understandings of the world or particular situations at hand. This perspective is of particular importance for robotics, because the formation of human-robot working partnerships or teams has the potential to combine radically different human and non-human perceptual and motor skills and abilities to form a more flexible team than a human team. However, a focus on building robots that are as humanlike as possible is likely to reduce the potential for combining radically different abilities in a human-robot team.

While not much talked about in social robotics, the tendency to value what communicators have in common over and above the different perspectives they might offer, has received considerable attention from communications scholars. In particular, John Durham Peters and Amit Pinchevski have critiqued communication theory that frames communication as a process of overcoming or eliminating difference, arguing that this idea is 'violent' to the other [41][42]. In making their arguments Peters and Pinckevski both draw upon the phenomenological tradition of communication theory that has its origins in the work of philosophers such as Levinas, who critiqued and extended Husserl's ideas. Fundamental to the phenomenological tradition, as developed by Levinas, is the idea that the other always retains a level of alterity and therefore cannot be completely comprehended by the self. From a Levinasian perspective, framing communication in terms of pairing and commonality, which are inherently reliant on the idea of understanding the other in terms of the self, results in the "reduplication of the self" (to use Peters' phrase) and the "elimination of difference" (the conclusion of Pinchevski) [41][42]. In terms of communication between humans, this violence is undesirable, because it can be linked with a disrespect for others, and their personal, cultural and social differences from the self.

In contrast, Levinas suggests that while self and other must come into proximity for communication to occur, the distance, and the related difference, that re- 
mains between them is the reason that communication occurs at all [37]. Levinas himself was concerned with exploring the ethics of human-human interactions, excluding animals and objects from his consideration as part of that focus[34][35]. However, as Christopher Diehm notes, aside from Levinas' insistence that only humans can take part in this type of ethical encounter, his philsophy in itself "does not require of the ethical relation an other who can reciprocate" [18]. Indeed, a number of scholars have argued that animals can take part in Levinasian encounters with humans [17][12], and David Gunkel has also considered the question of the moral agency of machines in light of Levinas' philosophy [27]. This paper sets asides the question of ethics in Levinas' self-other encounter to focus instead on the communicative potential it invokes. From this perspective the key elements are the self's decision to respond to the "call" of the other, alongside a decision to respect the presence of the other's absolute alterity from the self. As Levinas clarifies, the other need not communicate using language, but might express themselves using "the whole body" or "a hand or curve of the shoulder" [37]. It is therefore possible to consider the potential for human encounters with many different forms of robot by purposely extending Levinas' perspective on communication to include non-human others.

For social robotics, the critiques of commonality found in the work of communications scholars reinforce my suggestion that making robots like humans or animals, may reduce the possibilities of extending people's social relations by introducing them to new forms of robot that see and interpret the world differently from them. While encounters between humans and social robots, both humanlike and animal-like, attract and hold the attention of people during interactions, it is machinelike robots, such as those discussed below, that bring absolute alterity in form and communicative style into human-robot interactions. Extending Levinas' conception of communication to human-robot interactions emphasises the importance of the human's response to the robot, together with a constant acknowledgment of and respect for its difference. An analysis of the relations that build up between human team members and EOD robots supports this understanding of communication between humans and robots. These robots are certainly not designed to look like humans or animals, and yet they are accepted as valuable team members, with non-human characteristics that may be key to the operation of the team as a whole.

\section{Social connections between humans and machinelike robots in working teams}

Humans and machinelike robots work very closely together in EOD situations, with robots being deployed alongside humans in places such as Iraq and Afghanistan in recent years. Although the machines with which soldiers work are able to do certain tasks autonomously, such as righting themselves and retracing their steps when they lose radio contact with the team, they are generally under the constant control of a human operator. They are most often directed via radio signals using controllers very similar to those used to play computer games. EOD robots, such as PackBots and Talons, are not humanlike or animal-like, are not currently autonomous and do not have distinctive complex behaviours supported by artificial intelligence capabilities. They might therefore be expected to raise few critical issues relating to human-robot interaction, since communication with these machines relies on the direct transmission of information through radio signals, which have no emotional content and are not open to interpretation. Indeed, the fact that these machines are broadly not autonomous precludes them from being discussed as social robots according to some definitions [15]. In addition, the form of these machinelike robots clearly does not support an easy process of 'pairing', and their human-controlled movements would seem to offer little opportunity to reveal any level of 'harmonious behaviour' [31][11]. In spite of this, there is an increasing amount of evidence that EOD robots are thought of as team members, and are valued as brave and courageous in the line of duty [25][1][24][54][50]. It seems that people working with EOD robots, even though the robots are machinelike and under the control of a human, anthropomorphise and/or zoomorphise them, interpreting them as having individual personalities and abilities [24][54][50].

Clues to how such robots can become part of a closeknit team with humans, in spite of their lack of autonomy and seeming deficit of social and emotional expression, can be found in interviews with army personnel. It is clear that many EOD robots are assigned names and genders, Sgt. Talon for example being male, as was a robot called Frankenstein [25][50]. These robots are assigned status as individuals, in spite of the fact that there are only a certain number of makes and models of robot in use at any one time. This may be because, although radio controlled, the robots do reveal individual quirks and may make strange unexpected moves [25]. These individual differences are, of course, not an intentional part of the robot's design. They are not built in with programming nor do they emerge as learned re- 
sponses; instead, they are a consequence of the physical state of the machine and the environment in which it is working. Some of their actions may be related to software or hardware malfunctions, some to the extremes of environment in which they work and others to difficulties with maintaining clear radio contact. Finding the source of the robot's actions might seem important, since software and hardware glitches could be repaired, if identified. In practice, however, it seems that many small behavioural quirks remain over time, and it is the way that these individual behaviours are interpreted by operators and other soldiers that becomes important in assigning a personality to each robot.

EOD robots are read as expressive and communicative by their operators and other soldiers in ways that can be described in terms of what Erving Goffman calls "small behaviors" [26]. Goffman identifies these as "the glances, gestures, positionings, and verbal statements that people continually feed into the situation, whether intended or not" [26]. While EOD robots do not make verbal statements, their unintentional behaviours are read by human observers as "external signs of orientation and involvement" [26]. In terms of the communication theory I have already discussed, changes in the gaze direction of their camera, gestures of their arm and whole body movements of EOD robots are read as acts of non-verbal communication. For example, Sgt. Talon had a reputation as a reliable robot, and continued working even after "a couple of detonations in front of his face" [25]. The one occasion on which this robot broke down, the replacement that was sent in was destroyed, leading one of the EOD personnel to suggest that Sgt. Talon "shut down because he knew something bad would happen" [25]. It is difficult to consider these somewhat random and unplanned signals as precise transmissions of information. These robots only 'communicate' information directly through their responses to radio signalled instructions, and the readings provided by their sensors and the images captured through their cameras are also passed directly to their operators for interpretation. In spite of this, the unplanned and uncontrolled behaviours they reveal do seem to be read as persuasive communications, given the reactions they invoke in their human teammates.

It is particularly important to consider the effect of the socio-cultural positioning of EOD robots as lifesavers, sent into dangerous situations in lieu of a human soldier. As such, each time these robots complete a controlled explosion, defuse a bomb, or provide vital surveillance footage, their value rises and the bond felt with the robot will increase, alongside a sense of respect for and trust in the machine's abilities. EOD robots are not designed to be like humans or animals, but the re- lationship between operator and robot has nevertheless been compared with various kinds of human-dog relations, including with pets, hunting dogs and also working police dogs [46][50]. Ian Roderick argues, based on his detailed analysis of news reports and US Department of Defense press releases, that peoples' readings of non-autonomous EOD robots as agents stem from the social value that results from their positioning as saving people from injury or death, as opposed to from a mistaken impression that they are autonomous based on what he describes as a "proto-anthropological" process [46]. However, I would argue that the words that soldiers use to describe their robots demonstrate a level of anthropomorphism and/or zoomorphism, alongside a sense of valuing the robot for the danger it faces to keep humans safe. It is also worth noting that there is evidence that the foibles of some EOD robots leave human members of the team less well disposed towards them. Although these stories have not formed the basis of any news reports or research (to my knowledge) they are discussed in associated Reddit comment threads [51].

Staff engaged in repairing damaged robots may comment that a robot "came in missing half an arm ... but it's starting to look alive again", but they have a pragmatic response to the need to replace robots that have been damaged beyond repair [43][25]. However, soldiers deployed with robots on missions not only rely on the robot to save lives, but also interact with a particular robot closely over an extended period of time. Soldiers bringing in damaged robots for repair do not want to be given a new robot, they want the old one to be fixed, with one soldier giving a clear reason: "That little guy saved our butts on many occasions" [43][50]. For repair technicians, robots are understood as valuable team members that may even be "alive", but they are also machines that can be replaced, whereas, for the soldiers with which the robots are deployed, it is the individual machine that is meaningful [43]. It is the particular machine that is respected and trusted as a team member. In part, as Roderick suggest, this is likely to be because of the situation in which robot and human are embedded, placing the robot as a lifesaver [46]. However, an anthropomorphic response is also a presence, as one soldier stresses while discussing a robot named Frankenstein, "He was part of our team, one of us. He did feel like family" [25][50].

Although it may be difficult to understand these responses to robots when not involved in such situations in real life, the differences between soldiers' and engineers' appraisals of the importance of individual robots are mirrored in popular fiction when Luke Skywalker is offered a new R2 unit in replacement for his, which "seems a little beat up". Luke refuses, explain- 
ing: "Not on your life! That little droid and I have been through a lot together" before he turns to R2-D2 to ask "You okay, R2?" [38]. The parallel between responses to R2-D2 and EOD robots is clear; although, in contrast to EOD robots, R2-D2 is portrayed as an intelligent, autonomous machine, which might more easily be expected to elicit such loyalty from his human companion. However, in spite of their current lack of autonomy, EOD robots are able to bring non-human skills into the team by virtue of their size and maneuverability in constrained spaces, as well as the facility to add sensors that enable them to sense their surroundings based on heat signatures or chemical detection. As humans learn to work with particular robots, operating together over time as a team that completes joint tasks, a relation develops that increases the fluency of the team. The human operator becomes attuned to a few particular robots and is therefore better able to control those robots precisely given their individual behavioural quirks.

The examples above, relating to EOD robots, suggest that relations between humans and these robots develop in part because they are deployed into dangerous situations, in which the effect of positioning the robot as a lifesaver has a not inconsiderable influence. However, this type of response, where people begin to respect and trust a robot, has also been shown in experiments taking place in the safety of a laboratory with AUR, a robotic desk lamp created by Guy Hoffman at MIT. Unlike present day EOD robots, AUR is autonomous and acts on its sense of a human's vocal, hand, head and body movements, as well as its understanding of the joint task. A consideration of AUR therefore raises questions relating to how best to work with EOD robots, assuming that in the future these robots will be more autonomous in their operation as well as continuing to be positioned as saving lives.

\section{Experiments in working with a robot}

Hoffman designed and built AUR to take part in a series of experiments with human participants, where human and robot collaborated to complete a simple task. The experimental setup included three lecterns, the aim being for the human to direct the robot to turn towards a particular lectern and then change its lamp colour according to a written instruction hidden beneath one of a set of flaps on the lectern's surface. AUR was able to interpret simple voice commands, such as "come here", and could track the human's hand, head and whole body movements [30]. In this team, the human is valued for their overall comprehension of the task and the ability to pass instructions from the list they have been given to the robot, while AUR provides its non-human ability to cast coloured light alongside its responsiveness to movement and voice commands. The robot operated in two different ways. In the first, reactive mode, AUR simply followed the instructions and movements of the human; in the second, fluency mode, the robot learned the task at the same time as the human, and where possible began to anticipate the commands it was about to be given. AUR was developed to investigate ways of making human-robot interactions more fluent, as opposed to following strict turn-taking rules. Hoffman identifies anticipation, both about the surrounding environment and the actions of one's partner, as key in supporting successful humanrobot collaboration, something that is also identified, by Anthony Finn and Steve Scheding, as important for unmanned vehicle systems [30] [20]. Indeed, it seems reasonable to suggest that being able to anticipate both environmental situations and people's instructions might well be of importance for all social robots. In addition, Hoffman suggests that a process of "repetition, practice and rehearsal" is also a valuable support in improving the effectiveness of any collaborative partnership [30].

In reactive mode, people learnt to operate the robot, via movement, gesture and vocal commands and the robot simply does as instructed. The repetitious nature of the task therefore helped the human participant become adept at directing the robot accurately, but anticipation on the part of AUR did not come into play. In spite of this, people were still impressed by the lamp's ability to follow instructions. After working with the lamp over the course of the experiment, one participant noted that there were times when they felt as if they were "interacting with a being that was more alive... [than a] machine" [30]. This response is similar to that of EOD personnel and also repair technicians, who, even though they know the robot is under direct human control, nevertheless describe it as being "alive" [43]. AUR's intentions and attitudes were read by human participants based on the position of its supporting neck, lampshade head and light beam gaze [30]. In spite of the lamp's machinelike form (showing a close resemblance to a common household object), this robot was nonetheless read as somewhat humanlike and/or animal-like by participants. As was the case for EOD robots, its "small behaviors" place it as a social subject [26]. However, in reactive mode, communication with AUR is best assessed as a cybernetic process of information transfer, through which the human directs the robot, and the robot's response is implicitly communicated by its completion of the requested movement or change in lamp colour. The way in which the robot followed a command and then waited for the next instruc- 
tion made working with AUR in this mode very similar to the process of operating an EOD robot, although instead of a game-controller which might emphasise the nature of the relation, with AUR the free use of voice and gestures to command the robot may obscure the direct command structure.

The persuasive effect of AUR's behaviour, and the idea of the robot as a socially aware being and teammember, was much more marked in fluency mode. In this mode, the sequence of required moves and colours was learnt by both human and robot. The lamp was programmed to pay attention both to the human's instructions and to what it had learnt about the task for itself. This meant that the lamp gradually anticipated the human's movements and requests, fewer voice commands were required, and the team moved faster from one lectern to the next as the experiment progressed. In this mode, the sense that AUR was as committed to completing the task as the human was particularly well-communicated. Human and robot were working together in a way that set aside turn-taking, in favour of a more dynamic understanding in which they were part of a system of overlapping communicative acts of movement, together with occasional vocalisations and lamp colour changes. The importance of considering human and robot as communicating and working together as a system is also noted by those working with EOD teams: "We preach to everybody, you have to look at the system. Not just the machine, but the human" [25].

Hoffman designed AUR to look as much like a normal desk lamp as possible, seeking to avoid any anthropomorphic or zoomorphic interpretations. However, the comments of one participant clarify that they did anthropomorphise AUR, becoming "good friends" with the robot, such that they "high-fived mentally after the task was done" [30]. In contrast, another participant perceived AUR not "as human but ... possibly an animal" [30]. These examples, therefore, further support the contention that the human tendency to understand the unfamiliar by comparison with other more familiar things is unavoidable. In the case of AUR, as soon as it became clear that it was not an everyday lamp, people moved on to interpret it as somewhat humanlike or animal-like. Importantly, even as they did this AUR's difference from humans and animals remained obvious by virtue of its form and the robot's specific abilities. The same is true of EOD robots, and as such people's responses to these robots might be described as tempered anthropomorphism and/or zoomorphism.

\section{Tempered anthropomorphism and/or zoomorphism}

As I have already noted, processes of anthropomorphism and zoomorphism would seem flexible enough to cope with the presence of considerable difference, as opposed to relying on a close level of 'pairing' and 'harmonious behavior' [31][11]. Indeed, it seems that human understandings of robots, even those not designed to seem at all humanlike or animal-like, inevitably draw on anthropomorphic and/or zoomorphic responses. This is demonstrated by the examples I have discussed above, as well as in the way that people respond to robotic art installations [48]. Indeed, people may also read nonhumanoid robots in terms of something about which they have read, such as an alien in science fiction as noted in the video relating to the installation of $\mathrm{Au}$ tonomous Light Air Vessels (version 1.0), blimp-like robots that are exhibited in flocks [2]. It is reasonable to suggest that this type of response is unavoidable, being a pragmatic way for humans to understand anything that seems to be somewhat alive, by reading it as a little like another entity with which they have interacted before.

Rather than being a problem, a dangerous misreading of reality, such responses to robots are valuable, in particular where roboticists do not try to reinforce anthropomorphic and zoomorphic responses. For example, the designs of AUR and the various EOD robots demonstrate how successful human-robot interactions can be if one accepts the utility of such responses, alongside the way that they are tempered by a parallel clarity of understanding the robot as a machine. This type of tempering is also seen in our relations with other technologies such as cars and computers, which people may name and talk to as if they are human on some occasions, but which they also understand as machines that can be switched off, discarded, sold or recycled. This tempering, as the examples relating to EOD robots indicate, may be more challenging in the case of robots than cars or computers for a variety of reasons. As noted above, humans perceive EOD robots as individuals, value the non-human skills they bring to the team and are also working with them in dangerous situations which position the robot as a potential life saver. It may therefore be unsurprising that soldiers find it difficult to part with their robot, even if it is damaged beyond repair. However, it is also clear that soldiers are nonetheless able to send EOD robots into dangerous situations, in the same way that other circumstances may call for dogs and/or humans to be deployed. The relationship between humans and EOD robots is therefore a delicate balance between fostering 
a sense that the robot as an individual is important to the team, and the understanding that its 'life' is expendible in the context of the military setting in which it is being put to use.

The most important aspect of tempered anthropmorphism and/or zoomorphism is the way that it allows a robot to be considered familiar enough in its behaviour to interpret its movements as meaningful, while also leaving space to acknowledge its fundamental differences from both humans and animals. Although, as the early sections of this paper emphasised, roboticists who create social robots more often than not choose to reinforce an anthropomorphic or zoomorphic response, the more recent discussions of AUR and EOD robots demonstrate how not following this path can result in the development of flexible and resilient mid to long term relations between humans and machinelike robots. These relations are best understood to rely upon human understandings of the robot as somewhat humanlike or animal-like, but where these responses are continually temperered by also perceiving the robot as a machine. The machinelike nature of these robots is important because it offers the potential for them to share abilities that are very different from humans and animals when working in teams with humans. As I noted in the case of AUR, fluid communication between humans and machinelike robots may take some time to achieve, but the gradual development of the relation also allows time for the human to gain a sense of confidence in the abilities that the robot brings to the team. As David Bruemmer, cited by P. W. Singer, suggests, this level of confidence is a key part of enabling humans to develop a level of trust in the robots with which they work [50].

\section{Trust and respect in working teams}

The development of a working relation with robots such as EOD robots and AUR over time alters the way in which humans see the machine, from regarding it as an object, or a tool, to interpreting it as an intelligent member of the team. While this may seem reasonable in the case of AUR, at least when operating in fluency mode, it seems less well supported for the broadly radiocontrolled EOD robots. However, as I have shown, EOD robots are clearly adopted as team-members, both when on active duty and also during downtime, although the decision to take one robot fishing may simply indicate the need for humour when working in dangerous situations [25]. Most importantly, the relation that builds between human and robot is strong enough in the case of AUR, and in the case of EOD robots, for humans to develop a sense of "respect" for the robot and "trust" in its abilities [30][25]. Indeed, it is likely that people's feelings for EOD robots will only increase as their ability to operate autonomously is developed, even if there is always a point at which a human retains ultimate control. Although news reports, in particular those based on the recent research of Julie Carpenter, indicate a level of concern over the way in which people's feelings for EOD robots might affect their deployment, with the suggestion that favourite robots might be kept out of particularly dangerous situations even when best suited to act, there is no evidence that this occurs at present [1][24][54][10]. Rather than being worried by people's personalised response to a robot, I would argue that this is valuable in allowing human and robot to work together fluently as a team. At present, as I indicated earlier, the limited autonomy of EOD robots means that the increase in fluency is mostly related to the human becoming attuned to the robot's particular operating constraints and abilities. However, in the future, with increased autonomy, humans may need to be able to respond to the robot's own decisions to act based on its sense of the situation.

The development of feelings of trust and respect for robots will only become more important as robots themselves have more to offer in terms of their interpretation of a situation, and understanding of the best course of action. This is highlighted by considering a particular moment that occurred during human interactions with AUR. The human participant makes a mistake, and directs the lamp towards the wrong lectern. However, since it has its own understanding of the task, the lamp does not simply follow the human's lead, instead it does a 'double-take'. The lamp first turns the way it 'thinks' it has learnt is correct, and then turns to 'look' back at its human teammate. The movement is very small, but it is noticeable because it interrupts the flow of the system created as AUR and human move together through the experiment. The human sees the lamp's indecision, pauses and corrects their mistake to redirect the lamp towards the correct lectern, and then asks for the required colour [30]. For this response to occur, the human must build up a level of trust in and respect for the robot's point of view, such that they reassess their instruction and realise it was wrong. People working with AUR in a successful team are required to understand that the robot might remember how to complete the task better than they do, and in the future the same might be true of people working with intelligent EOD robots.

From a sociopsychological perspective, the lamp is able to persuade the human of its intelligence, such that the person accepts it knows best. Alternatively, taking a more phenomenological stance, through a process of 
tempered anthropomorphism the human recognises the robot's otherness, and judges its value as an individual with its own knowledge which may be better then their own. The importance of this momentary interruption in the flow of communication also emphasises the need to attend to individual communicators, even as the system of communication seems more important in such a dynamic interaction. Although roboticists such as Hoffman, and Finn and Scheding, are focused on the value of creating fluid and anticipatory communication in enabling humans and robots to work effectively together, the breaks in the system's flowing progress may be just as informative as the smooth interchange that surrounds them. Indeed, recognising those interruptions as valuable may well be key in enabling humans and robots to work together to the best of both their abilities, in particular as robots become increasingly autonomous.

\section{Conclusion}

The first half of this paper critically assessed some of the assumptions about form, behaviour and communication that are used to support the development of humanlike and animal-like social robots with the aim of destabilising the sense in which commonality is seen as a vital element of such designs. The idea that humans and machinelike robots can interact in effective and complex ways was then explored through an analysis of human relations with EOD robots, as well as human interactions with the robotic desk lamp, AUR. The ways in which humans talk about these machinelike robots indicate that the relations developed between human and robot are social in nature, as well as being directed towards the completion of particular tasks. Machinelike robots are perceived by people as communicative and collaborative as they work with them over time.

Key to the development of strong working humanrobot partnerships and teams is the presence of tempered anthropomorphic and/or zoomorphic responses in humans in relation to the robot. For machinelike robots, people's assessment of their behaviour as either somewhat humanlike or somewhat animal-like is constantly being reappraised in light of the fact that the robot is clearly a machine. This means that human interactions with machinelike robots retain a clear acknowledgement of the otherness of the robot at all times. This has the potential to allow a robot's different perceptual and motor skills to become a valuable part of a human-robot team or partnership within which humans and robots are able to work together to the best of their diverse abilities. Important to these relations is also the way that they develop over a period of time, during which human and robot work together, and may also learn together. The value of sharing experiences, even when a robot is not truly autonomous or able to learn for itself, as is the case with EOD robots, may be restricted to allowing humans to better operate a particular robot. However, in the future, if such robots make decisions about where to move or how to act based on their own sense of the environment and the task at hand, humans and robots will be in a more clearly collaborative relation, within which the human will need to respond to the robot based on the respect and trust that they have built up for that robot over time.

This paper's analysis of working relations between humans and machinelike robots as social could be extended to suggest that such robots have the potential to enrich people's lives more generally. Indeed, machinelike robots might be able to provide additional forms of social communication, as opposed to being overtly positioned as direct replacements for the social contact people might otherwise experience with humans or animals. Interactions between humans and robots that are not humanlike or animal-like in form have the potential to develop over time, leading to the formation of longterm relations within which the differences between participants are seen as an asset, as opposed to a difficulty that must be overcome.

Acknowledgements The completion of this paper was made possible by the receipt of a CCI Research Fellowship in the Centre for Culture and Technology at Curtin University.

\section{References}

1. Ackerman E (2013) Soldiers can get emotionally attached to robots, and that may not be a good thing. Spectr. IEEE 2. ALAVs Autonomous Light Air Vessels. http://www.alavs.com/. Accessed 20 April, 2014

3. Asimov I (1990) Robot visions. ROC, New York

4. Bartneck C, Chioke R, Menges R, Deckers I (2005) Robot Abuse - A Limitation of the Media Equation. Proceedings of the Interact 2005 Workshop on Abuse

5. Bekoff M (2007) The emotional lives of animals: a leading scientist explores animal joy, sorrow, and empathy-and why they matter. New World Library; Distributed by Pub. Group West, Novato, Calif.: [s.l.]

6. Breazeal C, Foerst A (1999) Schmoozing with robots: exploring the boundary of the original wireless network. Proc. Third Int. Conf. Cogn. Technol. pp 375389

7. Breazeal CL (2002) Designing sociable robots. MIT Press, Cambridge, Mass.

8. Capek K (2006) R. U. R. eBooks@Adelaide, The University of Adelaide Library, Adelaide

9. Carey J (1992) Communication as culture: essays on media and society. Routledge, New York

10. Carpenter J (2013) The Quiet Professional: An investigation of U.S. military Explosive Ordnance Disposal personnel interactions with everyday field robots. PhD, University of Washington 
11. Chang BG (1996) Deconstructing communication: representation, subject, and economies of exchange. University of Minnesota Press, Minneapolis

12. Clark D (1997) On Being the last Kantian in Nazi Germany: Dwelling with animals after Levinas. In: Ham J, Senior M (eds) Animal acts: configuring the humans in western history. Routledge, New York, pp 165198

13. Craig RT (1999) Communication theory as a field. Commun Theory 9:119161.

14. Dautenhahn K (2013) Human-Robot Interaction. In: Soegaard, Mads and Dam, Rikke Friis (eds) The Encyclopedia of Human-Computer Interaction, 2nd Ed. Aarhus, Denmark: The Interaction Design Foundation.

15. Dautenhahn K (1995) Getting to know each otherArtificial social intelligence for autonomous robots. Robot Auton Syst 16:333356. doi: 10.1016/0921-8890(95)00054-2

16. Dautenhahn K (1998) The art of designing socially intelligent agents: science, fiction, and the human in the loop. Appl Artif Intell 12:573617.

17. Derrida J (2002) The animal that therefore I am (more to follow). Critical Inquiry 28:369418.

18. Diehm C (2000) Facing nature: Levinas beyond the human. Philosophy Today 44:5159.

19. Ekmann P (1999) Basic emotions. Handb. Cogn. Emot.

20. Finn A (2010) Developments and challenges for autonomous unmanned vehicles: a compendium. Springer Verlag, Berlin

21. Flynn CP (2008) Social creatures: a human and animal studies reader. Lantern Books, New York

22. Fong T, Nourbakhsh I, Dautenhahn K (2003) A survey of socially interactive robots. Robot Auton Syst 42:143166.

23. Freud S (2004) The Uncanny (1919). In: Sandner D (ed) Fantastic literature: a critical reader. Praeger, Westport Conn., pp 74101

24. Garber M (2013) Funerals for Fallen Robots. The Atlantic

25. Garreau J (2007) Bots on the ground: In the field of battle (or even above it), robots are a soldier's best friend. Wash. Post

26. Goffman E (1972) Interaction ritual: essays on face-toface behaviour. Allen Lane, London

27. Gunkel DJ (2012) The machine question: critical perspectives on AI, robots, and ethics. MIT Press, Cambridge, Mass

28. Hanson Robotics Vision. In: Hanson Robotics. http://hanson.robotics.com/. Accessed 18 Oct 2008

29. Hearne V (2000) Adam's task: calling animals by name. Akadine Press, New York

30. Hoffman G (2007) Ensemble: fluency and embodiment for robots acting with humans. Ph.D., Massachusetts Institute of Technology

31. Husserl E (1973) Cartesian Meditations: An Introduction to Phenomenology. Martinus Nijhoff, The Hague

32. Lang F, von Harbou T (1927) Metropolis.

33. Leite I, Martinho C, Paiva A (2013) Social Robots for Long-Term Interaction: A Survey. Int J Soc Robot 5:291308. doi: $10.1007 / \mathrm{s} 12369-013-0178-y$

34. Levinas E (1990) The name of a dog, or natural rights. Difficult freedom. The Athlone Press, London, pp 151153

35. Levinas E (1989) Is ontology fundamental? Philosophy Today 33:121129.

36. Levinas E (1989) The Other in Proust. In: Hand S (ed) Levinas Read. Blackwell Pub., Oxford, pp 160165

37. Levinas E (1969) Totality and infinity. Duquesne University Press, Pittsburgh

38. Lucas G (1977) Star Wars Episode IV: A New Hope.
39. Menzel P, D'Aluiso F (2000) Robo sapiens: evolution of a new species. MIT Press, Cambridge, Mass.

40. Mori M (1970) The uncanny valley. Energy 7:3335

41. Peters JD (1999) Speaking into the air: a history of the idea of communication. University of Chicago Press, Chicago; London

42. Pinchevski A (2005) By way of interruption: Levinas and the ethics of communication. Dusquene University Press, Pittsburgh, Pennsylvania

43. QinetiQ (2009) Fast, powerful and versatile, high payload robot technology. TALON. QinetiQ, Farnborough

44. Reeves B, Nass CI (1996) The media equation: how people treat computers, television, and new media like real people and places. CSLI Publications; Cambridge University Press, Stanford, Calif.: New York

45. RoboKind About Us. In: RoboKind Website http://www.robokindrobots.com/. Accessed 18 Dec 2013

46. Roderick I (2010) Considering the fetish value of EOD robots: How robots save lives and sell war. Int J Cult Stud 13:235253. doi: $10.1177 / 1367877909359732$

47. Russell JA (1994) Is there universal recognition of emotion from facial expressions? A review of the cross-cultural studies. Psychol Bull 115:102141. doi: 10.1037/00332909.115.1.102

48. Sandry E (2012) Dancing around the subject with robots: ethical communication as a "triple audiovisual reality". Platf J Media Commun 4:7990.

49. Short S (2003) The measure of man?: Asimov's Bicentennial Man, Star Trek's Data, and being human. Extrapolation 44:209223.

50. Singer PW (2010) Wired for war: the robotics revolution and conflict in the twenty-first century. Penguin Books, New York

51. The Real Opie (2013) Soldiers are developing relationships with their battlefield robots, naming them, assigning genders, and even holding funerals when they are destroyed. In: Reddit/r/technology. http://www.reddit.com/r/technology. Accessed 18 Dec 2013

52. Turkle S (2005) The second self: computers and the human spirit, MIT Press Edition. MIT Press, Cambridge, Mass.

53. Turkle S, Breazeal C, Dast O, Scassellati B (2006) First encounters with Kismet and Cog. In: Messaris P (ed) Digit. Media Transform. Hum. Commun. Peter Lang, New York, pp 303330

54. Waldman K (2013) Are soldiers too emotionally attached to military robots? Slate

Eleanor Sandry is a Lecturer in the Department of Internet Studies at Curtin University, Western Australia. Her research is focused on exploring a diverse range of communication theory in developing an ethical and pragmatic recognition of, and respect for, otherness and difference in communication. 
This version is the author's accepted manuscript. A few corrections were made before final publication at: http://link.springer.com/article/10.1007/s12369-014-0278-3

In the main these were fixes for small typographic errors and also minor wording changes for readability.

The following errors were also fixed:

First paragraph of section 2 citation should be to [3,49]

Fifth paragraph of section 5 citation should be to $[36,37]$

Errors in reference list for Ekmann should read:

Ekman P (1999) Facial expressions. In: Dalgleish T, Power T (eds) The Handbook of Cognition and Emotion. Wiley, Chichester, pp 301-320

Full reference for Lang F, von Harbou T (1927) Metropolis. Universum Film (UFA) 